\title{
EL LATIDO ELECTRÓNICO AFRICANO EN LA SEGUNDA DÉCADA DEL SIGLO XXI ${ }^{1}$
}

\section{AFRICAN ELECTRONIC HEARTBEAT IN THE SECOND DECADE OF THE 21ST CENTURY}

\author{
ALBERTO FLORES GALÁN \\ Museo Vostell Malpartida
}

Recibido: 21/04/2019 Aceptado: 29/09/2019

\section{RESUMEN}

La investigación analiza un conjunto de propuestas electrónicas surgidas en la segunda década del siglo XXI que están definidas por la absorción de influencias africanas de diverso tipo.

Palabras clave: Shangaan electro, gqom, singeli, balani show, Príncipe, Bamako, Dar es-Salam, Durban, Lisboa.

\section{ABSTRACT}

The research addresses a number of electronic proposals from the second decade of the 21 st Century that absorb a wide range of African genres.

Keywords: Shangaan electro, gqom, singeli, balani show, Príncipe, Bamako, Dar es Salaam, Durban, Lisbon.

1 Este texto ha sido posible gracias a la Ayuda del S.E.C.T.I. (Sistema Extremeño de Ciencia y Tecnología e Innovación) al Grupo de Investigación de la Junta de Extremadura "Arte y Patrimonio Moderno y Contemporáneo (HUM012)”. 
En los primeros lustros del siglo XXI los universos del arte sonoro y la música de vanguardia han experimentado algunos cambios sensibles, cuando no revolucionarios. En lo que se refiere a la creación electrónica que hunde sus raíces (y cables) ${ }^{2}$ en la tradición del continente negro una asombrosa variedad de músicas electrónicas han surgido de toda África en los últimos quince años ${ }^{3}$. De entre esta enorme diversidad (que oscila entre el kuduro angoleño, el mahraganat egipcio o el viaje de ida y vuelta realizado por el hip-hop, que en cierto modo regresó a la tierra y a la tradición oral de los griots) el estudio analiza aquellas propuestas que han sido celebradas en ámbitos especializados en el análisis de la experimentación sónica. Nos centramos en los géneros shangaan electro y gqom (ambos de origen sudafricano), las experiencias de Nyege Nyege Tapes en Uganda y Sahel Sounds en África Occidental y, ya en territorio europeo, la aventura de arte y vida del sello discográfico portugués Príncipe Discos.

Para contextualizar la importancia de la absorción de influencias africanas de muy diverso tipo por parte de estos artistas resulta oportuno mirar en primer lugar al espejo retrovisor. Con este propósito acudimos a las palabras de Luis Lles, que en un contexto de revisión de lo acaecido en la última década del siglo pasado, por un lado afirmaba que la música popular moderna del continente seguía entonces su curso sin dejar demasiado cauce a la necesaria renovación ${ }^{4}$ $\mathrm{y}$, por otro -haciendo ya referencia a la producción electrónica- argumentaba: si hacemos un simil con las artes plásticas, se podría decir que hemos pasado la época de las vanguardias históricas, que han terminado los tiempos modernos y que ya está aqui la posmodernidad dance, la era del "revival" y el reciclaje ${ }^{5}$. Con la perspectiva que produce el paso del tiempo, es posible confirmar a día de hoy lo apuntado por Jaron Lanier en su manifiesto "Contra el

2 Las lianas se convierten en cables electrónicos y los tambores son propulsados al espacio exterior. En: LLES, L., “Afro-beat. Un safari por el África electrónica”, en: Dance de Lux 2000, Ediciones RDL/Rockdelux, Barcelona, 2000, p. 71

3 RAVENS, C, "Plug and Play", en: The Wire n 400, Londres, Junio 2017, p. 31.

4 La música moderna africana tuvo su gran momento de auge en la segunda mitad de los años ochenta. La excitación que produjo entonces escuchar el latido tribal y las voces escalofriantes del África ancestral en perfecta convivencia con las guitarras eléctricas y los sintetizadores no ha podido ser superada en los años noventa, década en la que sólo las producciones de Youssou N'Dour, Manu Dibango, Angélique Kidjo, Mory Kante y, sobre todo, el senegalés Baaba Maal han logrado explorar la contemporaneidad electrónica (algunos de ellos cuentan con excelentes remezclas dance) y aproximarse a una sonoridad del siglo XXI. [...] El soukous, la kwuela, el jive, el hi-life, la juju music y toda esa galaxia de ritmos de nombres exóticos que intentamos aprender a pronunciar en los años ochenta siguen su curso sin dejar demasiado cauce a la necesaria renovación. En: LLES, L., "Agitando los 90. Estilos: World Music", en: Rockdelux n 164, Ediciones RDL/Rockdelux, Barcelona, Junio 1999.

5 LLES, L., "Nuevos estilos", en: Dance de Lux 1999, Ediciones RDL/Rockdelux, Barcelona, 1999, p. 55. 
rebaño digital", en el que se afirma que Internet ha imposibilitado a la cultura popular establecer estilos musicales realmente nuevos y definidos. En este sentido, el escritor e informático estadounidense señala que el gangsta rap no existía en los años sesenta [...] y que el heavy metal no existía en los cuarenta ${ }^{6}$ y lamenta que todo es retro, retro, retro ${ }^{7}$ en un periodo de nuestra historia denominado por Simon Reynolds como la "época YouTube Wikipedia Rapidshare iTunes Spotify" y en el que la distancia y la demora han sido reducidos a prácticamente nada ${ }^{8}$.

Lo último que sucedió antes que Internet y las redes sociales pasaran a ser la plataforma dominante para la música y la cultura emergentes ${ }^{9}$ fue el grime, un sonido surgido de los barrios de viviendas sociales del este de Londres (particularmente Tower Hamlets, London Borough of Newham, Barnet y Hackney) que acabaría definiendo la música electrónica de la siguiente década. El grime - cuyo territorio central no supera las escasas millas cuadradas de una parte del este de Londres a las que no alcanza el servicio de metro ${ }^{10}-$ germinó en radios piratas y comunitarias, especialmente Rinse FM, Deja Vu FM o Mystic FM, que entre 1999 y 2004 sentaron las bases de la música underground londinense por excelencia ${ }^{11}$. Se trató de un género insular que pudo disponer de tiempo para desarrollarse con cimientos sólidos.

Las casi dos décadas transcurridas desde entonces nos sitúan en un contexto bien diferente, aunque los mismos playstation, ritmos baratos o Fruity Loops ${ }^{12}$ que posibilitaron el nacimiento del grime han resultado ser un aliado esencial para iniciativas contemporáneas como el gqom de Durban, el singeli de Dar es-Salam o la batida afro-portuguesa dada a conocer por Príncipe. En referencia a esta última, Rory Gibb apunta en su reseña en la revista británica The Wire del maxi de debut de Tia Maria Produções - "Tá Tipo Já Não Vamos Morrer" (Príncipe, 2014) - que ambos géneros han sido creados por jóvenes productores y DJs de áreas marginadas que utilizan software barato y estropeado para facturar embriagadora nueva música lo-fi de baile ${ }^{13}$. La

6 LANIER, J., Contra el rebaño digital. Un manifiesto, Debate, Barcelona, 2011, p. 167.

7 Op. Cit, p. 166.

8 REYNOLDS, S., Retromania. Pop Culture's Addiction to it's own Past, Faber \& Faber, Londres, 2012, p. 80.

9 COLLINS, H., This is Grime. Hodder \& Stoughton, Londres, 2016, p. 173.

10 REYNOLDS, S., "Grime", en: Rob Young (ed), The Wire Primers. A Guide to Modern Music, Verso, Londres, 2009, p. 78.

11 HANCOX. D., "Grimetapes. Online Pirate Hoard", en: The Wire n 298, Londres, Diciembre 2008, p. 12.

12 COLLINS, H., This is Grime. Hodder \& Stoughton, Londres, 2016, p. 40.

13 GIBB, R., Reseña de "Tá Tipo Já Não Vamos Morrer", de Tia Maria Produções, en: The Wire $\mathrm{n}^{\circ} 369$, Londres, Noviembre 2014. 
producción del primer grime es realmente pobre y Fruity Loops es otro punto de contacto con el sonido de los jóvenes productores del extrarradio de Lisboa, que ha sido definido por Robert Barry como estrepitosos ritmos Fruity Loops y ondas cuadradas sincopadas ${ }^{14}$.

Príncipe ha propiciado que desde los bairros sociais de Lisboa que históricamente han acogido a comunidades con raíces en Angola, Mozambique, Cabo Verde o Guinea-Bissau se desarrolle una de las escenas de club emergentes más emocionantes del mundo ${ }^{15}$. Una comunidad muy heterogénea de artistas, cuyos nombres más conocidos son DJ Marfox, Nídia, DJ Firmeza y DJ Nigga Fox, ha entusiasmado a oyentes internacionales [...] por la sensación de estar escuchando un estilo al que se le ha permitido evolucionar en un relativo aislamiento ${ }^{16}$. Porque en efecto este género propio que reformula de un modo singular la herencia patrimonial del kuduro, la tarraxinha y otros estilos electrónicos de raíz africana y lusófona había permanecido oculto a plena vista ${ }^{17}$ hasta que fue descubierto por una compañía discográfica que también tiene su marco referencial en la experimentación sonora y las artes: creemos necesario establecer que entre los responsables del sello lisboeta destacan Nelson Gomes (de Gala Drop), André Abel (integrante de Tropa Macaca, junto a la artista visual Joana da Conceição) o el artista plástico Márcio Matos.

Capítulo aparte merece el estilo sudafricano conocido como shangaan electro, que va a colocarse en el primer plano del relato de la música electrónica africana en 2010, cuando el sello Honest Jon's Records edita la recopilación "Shangaan Electro: New Wave Dance from South Africa". En rigor, se trata de un retorno a la tradición a través de la tecnología ${ }^{18}$, pues se combina con asombrosa naturalidad la música tradicional de los townships sudafricanos con tsonga disco, kwaito y house. El productor Nozinja (a.k.a. Zinja Hlungwani), figura central de la escena, es el principal responsable de la popularización de un estilo reconocible por su extraordinaria velocidad (180 BPM) que ha sido denominado como música frenética afro-futurista ${ }^{19}$. Bajo el paraguas de Nozinja Music Productions, y antes de este reconocimiento internacional, se

14 BARRY, R., Reseña del EP recopilatorio "Cargaa 1" (Warp, 2015) y del EP "Noite e Dia (Príncipe, 2015), de DJ Nigga Fox, en: The Wire no 375, Londres, Mayo 2015, p. 46.

15 BARKER, S., Reseña de "Chapa Quente", de DJ Marfox, en: The Wire n 387, Londres, Mayo 2016, p. 59.

16 RAVENS, C., "Discos Inferno. Lisbon Label Príncipe Discos is an International Channel for the City's Batida Underground", en: The Wire n 377, Londres, Julio 2015, p. 15.

17 Op. cit.

18 BOON, M., Reseña de "Shangaan Electro: New Wave Dance from South Africa", en: The Wire ${ }^{\circ} 318$ Londres, Agosto 2010, p. 63.

19 Op cit. 
realizaron competiciones en plena calle y numerosos casetes y discos com-pactos de Tshetsha Boys, Tiyiselani Vomaseve o el propio Zinja Hlungwan se distribuyeron ampliamente por Sudáfrica durante años. Años más tarde, en 2015, el sello británico Warp edita "Nozinja Lodge", primer larga duración de Nozinja en solitario para el mercado global.

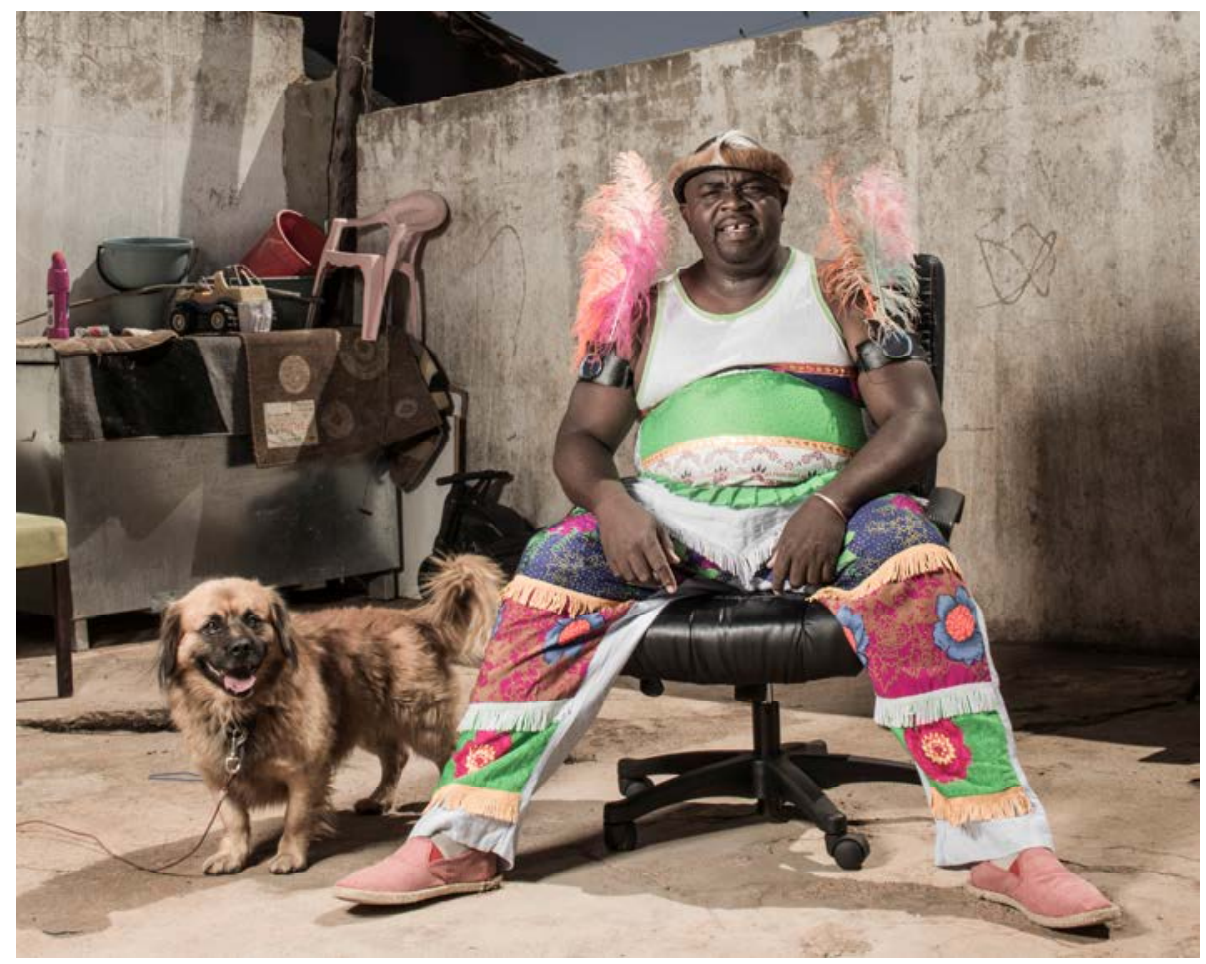

Nozinja, 2015. Fotografía: Chris Saunders

En una órbita similar hemos de ubicar los balani shows desarrollados en estos mismos años en Bamako y dados a conocer en occidente por el estadounidense Christopher Kirkley, cuyo sello Sahel Sounds también ha publicado la serie de recopilaciones "Music from Saharan Cellphones". Con una puesta en escena heredera de los sound systems en Jamaica, Balani Show presenta una nueva música electrónica maliense, conocida como ambience, deudora tanto del coupé decalé como de la música tradicional del país interpretada con balafón. De hecho, balani viene a significar "pequeño balafón" y su surgimiento puede considerarse como una consecuencia de la dificultad sobrevenida en las fiestas organizadas tradicionalmente: llegó un momento en el que contratar a 
intérpretes de balafón resultaba tan costoso que se acudió a samplers de grabaciones en casete sobre las que se añadieron ritmos electrónicos actuales. Los artistas más destacados de esta redefinición de la tradición musical de Mali que abraza la tecnología lo- $f i$ son, entre otros, DJ Sandji, DJ Senateur, DJ Balani, Kaba Blon y Mamelon ${ }^{20}$.

Más recientemente, un nuevo micro-género de música de baile ha surgido de los dormitorios y bolsillos traseros ${ }^{21}$ de adolescentes y jóvenes de los townships de Durban, en Sudáfrica. Conocido con el término de gqom, una palabra zulú tomada del nombre de tambores utilizados en música tradicional ${ }^{22}$, el estilo resulta atractivo tanto por ofrecer un sabor más rural como por su carácter $l o-f i$, siendo muy frecuente (como sucede en el primer grime o las ya citadas producciones inaugurales de Príncipe Discos) la utilización del software Fruity Loops. Acreditado en Europa gracias a la labor del sello discográfico Gqom Oh!, dirigido por el italiano Nan Kolè, el género fue ampliamente reproducido en teléfonos móviles en Durban y aún hoy la plataforma sudafricana de streaming y descarga KasiMP3 es el mejor método de promoción "en casa" de productores como Dominowe, Emo Kid, Mafia Boyz, Forgotten Souls o DJ Lag.

Y ya en el año 2017, la labor desarrollada por el sello Nyege Nyege Tapes -dirigido por el belga Derek Debru y el griego-armenio Arlen Dilsizian, ambos residentes en Kampala ${ }^{23}$ - ha puesto de relieve micro-géneros de África Oriental como el electro acholi (cuyo principal exponente es el ugandés Otim Alpha) o, particularmente, el singeli surgido de los estudios Sisso de la megalópolis tanzana de Dar es-Salam. Este último estilo ha sido definido como una de las más estimulantes (y menos adaptables) nuevas reiteraciones del folclor electrónico africano ${ }^{24}$ y puede llegar a alcanzar los 200 BPM. Sus principales exponentes son, entre otros, Bwax, Bampa Pana, Dogo Niga, Abbas Jazza o Sisso.

20 KIRKLEY, C., "Global Ear. Bamako”, en: The Wire n 342, Londres, Agosto 2012, pp. 16 y 17.

21 RAVENS, C., Reseña del doble álbum recopilatorio "Gqom Oh! The Sound of Durban Vol 1", en: The Wire no 383 Londres, Enero 2016, p. 65.

22 SAWERS, C., "Gqom with me", en: The Wire no 395, Londres, Enero 2017, p. 16.

23 HUTCHINSON, K., "Global Ear. Jinja", en: The Wire n 418, Londres, Diciembre 2018, pp. 16 y 17.

24 RAVENS, C., Reseña del disco "Poaa", de Bamba Pana, en: The Wire no 417, Londres, Noviembre 2018, p. 66. 


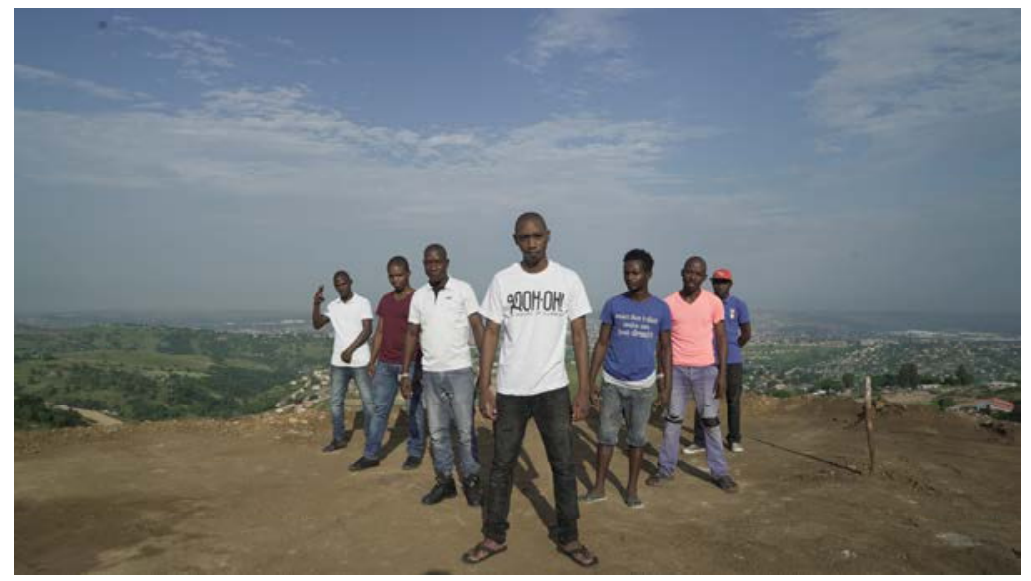

Emo Kid feat. WorstHood, de Gqom Oh! Fotografía: Tommaso Cassinis para Crudo Volta

Como conclusión, los géneros estudiados no solo defienden una vorágine polirrítmica y sincopada desprovista de cualquier envoltorio exótico - recurriendo, por tanto, a la esencia misma de la música africana ${ }^{25}$ - sino que lo hacen manteniendo intacta la autenticidad de sus propuestas. La aparición de estos estilos ha generado la misma sensación de extrañeza que suscitó la aparición de los primeros discos de grime hace más de tres lustros. Por último, una cuestión bien diferente - que requeriría un estudio aparte - es el análisis de la plausible contribución de sellos europeos o estadounidenses. Son estos quienes están posibilitando, aún más que la tecnología digital, una exposición global al desarrollo de géneros híbridos muy claramente definidos por parámetros psico-geográficos.

\author{
Alberto Flores Galán \\ Museo Vostell Malpartida \\ Carretera de Los Barruecos, $\mathrm{s} / \mathrm{n}$. \\ 10910 Malpartida de Cáceres (Cáceres) \\ albertofg.mvm@juntaex.es
}

25 El senegalés Youssou N'Dour señalaba, al inicio de la última década del siglo pasado, dos tergiversaciones recurrentes de Occidente sobre la música popular de África: por un lado un deseo [...] de amontonar toda la música africana en una única categoría cuyo rasgo distintivo es simplemente la procedencia geográfica y por otro una preferencia casi automática de algunos occidentales por elementos "tradicionales" africanos. N'DOUR, Y., "World Music' or a world of musics? An African Perspective", en: Worldwide. Ten Years of WOMAD, WOMAD Communications / Virgin Records, Londres, 1992, p. 65. 
